\title{
Metastasis of duodenal adenocarcinoma to the urinary bladder presenting as hematuria
}

\author{
Rani Tulsi ${ }^{1}$, Muhammad Manzoor Ul Haque ${ }^{1}$, Farina M Hanif ${ }^{1}$, Asha Devi ${ }^{1}$, \\ Muhammed Mubarak ${ }^{2}$, Nasir Hassan Luck ${ }^{1}$ \\ 'Department of Hepatogastroenterolgy, Sindh Institute of Urology and Transplantation (SIUT), Karachi, Pakistan; \\ 2Department of Histopathology, Sindh Institute of Urology and Transplantation (SIUT), Karachi, Pakistan
}

\section{TO THE EDITOR}

Signet ring adenocarcinoma of urinary bladder is a rare condition. Most cases reported in literature are primary and only one case of metastatic signet ring carcinoma has been reported. ${ }^{[1]}$ This tumor has poor response to chemotherapy and radiotherapy, and is associated with dismal prognosis. ${ }^{[2]}$ Here, we report a case of primary duodenal signet cell adenocarcinoma metastasizing to urinary bladder which is exceptionally rare and only one case is previously reported in literature. ${ }^{[3]}$

A 25-year-old man, presented in urology clinic with complaints of gross hematuria and urinary urgency for 3 months. In addition, he had associated complaint of loss of appetite, generalized weakness, unintentional and undocumented weight loss. He had no past medical history of note, while in past surgical history, he underwent extracorporeal shock wave lithotripsy (ESWL) and pyelolithotomy for left renal stone. On physical examination, he was a young male with Eastern Cooperative Oncology Group (ECOG) Performance Status of 2 having average height and thin built. His anterior cervical lymph nodes were palpable bilaterally, 1-2 cm in size, hard in consistency and nontender to touch, while rest of the systemic examination was unremarkable.

His initial investigation showed hemoglobin of $13.1 \mathrm{~g} / \mathrm{dL}$, white blood cell count of 6.4 $\times 10^{\circ} / \mathrm{L}$, platelet count $236 \times 10^{9} / \mathrm{L}$, urea $44 \mathrm{mg} / \mathrm{dL}$, serum creatinine $1.88 \mathrm{mg} / \mathrm{dL}$, prothrombin time 12.8 seconds, and international normalized ratio 0.94 . Ultrasound abdomen revealed minimal fullness of right kidney with prominent ureter along with hydronephrosis of left kidney with cortical thinning. Subsequently, for persistent hematuria, he underwent cystoscopy that showed a large growth on the right lateral wall with small capacity urinary bladder and multiple strictures in the urethra. Multiple biopsies of urinary bladder growth were taken. Microscopic examination showed a neoplastic lesion exhibiting sheets of signet ring cells with abundant clear cytoplasm and eccentrically placed hyperchromatic nuclei (Figure 1A). These cells showed positivity for mucin on AB-PAS-D stain (Figure 1B). Tumor was found to invade the underlying stroma. Detrusor muscle was not involved. IHC markers with appropriate positive controls were applied and CK7, CK 20, CK 19, CDX2, Villin, and $\beta$-Catenin were positive. Histological features were suggestive of poorly differentiated signet ring cell adenocarcinoma, likely of gastrointestinal origin

To rule out primary site, he underwent upper and lower gastrointestinal endoscopy, which showed shallow duodenal ulcer, while rest of mucosa was normal and biopsy of duodenal ulcers revealed poorly differentiated signet ring cell adenocarcinoma with similar marker positivity to that of urinary bladder growth.

To stage the disease contrast, enhanced computed tomography (CT) scan of chest, 


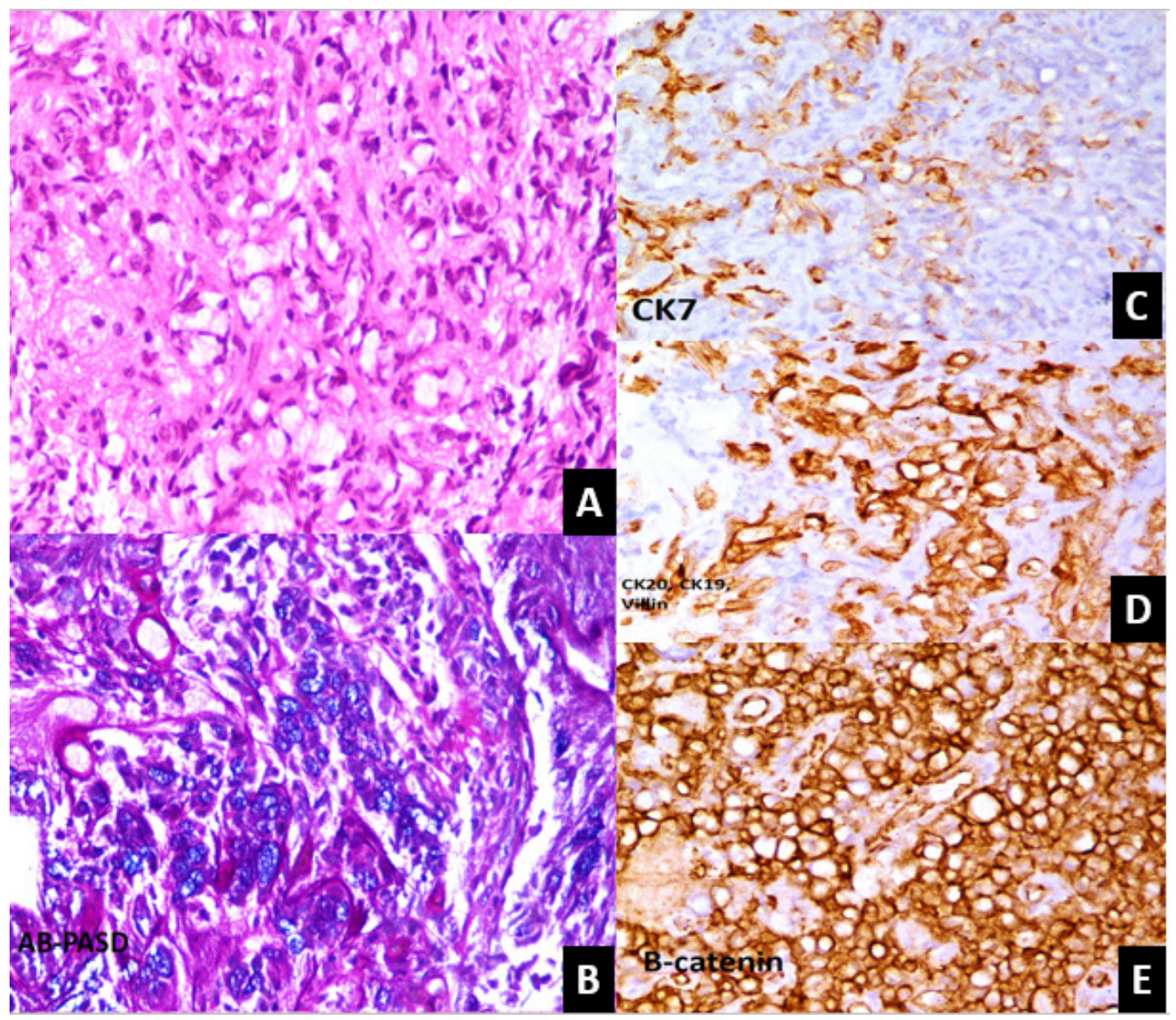

Figure 1: Microscopic examination of Bladder Growth Biopsy. A: Sheets of signet ring cell exhibiting abundant clear cytoplasm with eccentrically placed nuclei. B: Cells showing positivity for mucin in Ab-PASD stain. C: Tumor cells show focal positivity for CK 7. D: Tumor cells show positivity for CK 19 , CK 20 and villin. E: Diffuse cytoplasmic positivity for Beta catenin.

abdomen, and pelvis was performed, which revealed an irregular heterogeneously enhancing soft tissue density mass lesion diffusely involving the urinary bladder predominantly right lateral wall. It was also involving right ureterovesical junction and distal part of right ureter. Its infiltration into right seminal vesicle was also seen. Right kidney measured $12.2 \mathrm{~cm} \times 7.3 \mathrm{~cm}$, while left measured $3.8 \mathrm{~cm} \times 2.7 \mathrm{~cm}$. Multiple enlarged abdominopelvic lymph nodes were seen, few of them were necrotic, largest $2.2 \mathrm{~cm} \times 2.1 \mathrm{~cm}$ in paraaortic region and bilateral inguinal region. For obstructive uropathy, bilateral percutaneous nephrostomy tube was placed. Oncology team was taken on board, and considering the patient's poor performance status, chemotherapy could not be offered. He was advised palliative care and patient died after one month of diagnosis.

Urinary bladder carcinomas is the $9^{\text {th }}$ commonest malignancy that is seen around the world, with urothelial carcinoma being the main histological subtype. ${ }^{[4]}$ Metastatic carcinomas of the urinary bladder account for about less than $2 \%$ of all the bladder tumors and present mostly after wide spread dissemination. ${ }^{[5,6]}$ Adenocarcinoma of the urinary bladder makes $0.5 \%-2 \%$ of the bladder carcinoma in the United States and may be primary or secondary in origin, with the latter being the more common subtype. ${ }^{[7]}$

Secondary adenocarcinoma is either due to direct extension or from a distant site metastasis, with the main sites of origin being the prostate, the cervix, the colon, the lung, and the breast. These may spread directly or via the hematogenous or the lymphatic route. For the correct diagnosis, the histological findings need to be correlated with the history along with the immunohistochemical markers. The main cause of secondary bladder metastasis is the colorectal adenocarcinoma and may show finger like projections similar to the bladder villous adenoma.

Small intestine carcinomas, even though rare, mostly metastasize to liver and regional lymph nodes. Their metastasis to the urinary bladder has sporadically been reported in the literature. Morikawa et al. ${ }^{[3]}$ showed a solitary urinary bladder metastasis from a small intestine carcinoma, with the patient having urological 
symptoms. This was diagnosed based upon histology and immunohistochemical analysis.

In conclusion, we report the second case of duodenal adenocarcinoma metastasizing to urinary bladder, reported so far in the literature.

\section{Conflict of Interest}

None declared.

\section{REFERENCES}

1. Silva RD, de Araujo GN, Hommerding CCL, Censi KC, Garcia SP, et al. Signet-Ring Cell Gastric Carcinoma Metastatic to Bladder. J Clin Case
Rep 2015; 5:559.

2. Erdogru T, Kiligaslan I, Esen T. Primary signet ring cell carcinoma of the urinary bladder. Review of the literature and report of two cases. Urol Int 1995; 55: 34-7.

3. Morikawa T, Goto A, Nishimatsu H, Fukayama M. Metastatic Small Intestinal Cancer of the Urinary Bladder. Case Rep Oncol 2010; 3: 334-8.

4. Wong MCS, Fung FDH, Leung C, Cheung WWL, Goggins WB, Ng CF. The global epidemiology of bladder cancer: a joinpoint regression analysis of its incidence and mortality trends and projection. Sci Rep 2018; 8: 1129 .

5. Melicow MM. Tumors of the urinary bladder: a clinico-pathological analysis of over 2,500 specimens and biopsies. J Urol 1955; 74: 498-521.

6. Ganem EJ, Batal JT. Secondary malignant tumors of the urinary bladder metastatic from primary foci in distant organs. J Urol 1956; 75: 965-972.

7. Dadhania V, Czerniak B, Guo CC. Adenocarcinoma of the urinary bladder. Am J Clin Exp Urol 2015; 3: 51-63.

How to cite this article: Tulsi R, UI Haque MM, Hanif FM, Devi A, Mubarak M, Hassan Luck N. Metastasis of duodenal adenocarcinoma to the urinary bladder presenting as hematuria. J Transl Intern Med 2021; 9: 143-5. 